\title{
Microwave and Hard X-ray Imaging of Energetic Electrons in Solar Flares: Event of 2003 June 17
}

\author{
M. R. Kundu ${ }^{1}$, E. J. Schmahl ${ }^{1,2}$ and S. M. White ${ }^{1}$ \\ ${ }^{1}$ Department of Astronomy, University of Maryland, College Park MD 20742 \\ ${ }^{2}$ NASA Goddard Space Flight Center, Greenbelt, MD 20771
}

\begin{abstract}
We discuss a large flare that was observed simultaneously by RHESSI in hard X-rays and by the Nobeyama Radio Heliograph (NoRH) in microwaves. The imaging observations made both by RHESSI and NoRH show many interesting features which may be relevant for producing realistic flare models.
\end{abstract}

Keywords. Sun: flares, radio radiation, X-rays, gamma rays

\section{Introduction}

We discuss how one large flare may be used to investigate flare models using simultaneous observations obtained with two instruments - the Nobeyama Radio Heliograph (NoRH) at 17 and $34 \mathrm{GHz}$, and the Reuven Ramaty High Energy Solar Spectroscopic imager (RHESSI). As we know energetic electrons accelerated to nonthermal energies are seen via microwave and hard $\mathrm{X}$-ray emission from the solar corona. Imaging observations are necessary to study the energetic electrons in events such as these. NoRH measures both Stokes I and V at $17 \mathrm{GHz}$, and at $34 \mathrm{GHz}$ Stokes I alone, with good sensitivity and spatial resolution of 12" and 8" arc seconds, respectively at the two frequencies. Hard X-ray emitting electrons are mapped by RHESSI.

Nonthermal microwave emission during large solar flares is produced by gyrosynchrotron mechanism which involves coronal magnetic fields of at least a few hundred gauss and electrons of energy of a few hundred $\mathrm{keV}$. Hard X-ray emission, on the other hand, is produced by bremsstrahlung from beamed or trapped electrons of a few to hundreds of keV energy. The two different methods of mapping energetic flare electrons therefore complement each other, and provide good means of testing flare models which have been abundant in the recent literature. The RHESSI HXR and NoRH microwave imaging observations of the flare of 2003 June 17 are used here as an example. The flare in question was of class M6.8, and it was observed in AR 10386, a beta-gamma-delta region, two days after its east-limb appearance.

In this event, during the pre-impulsive and early impulsive rise phases, we see brightening at low (3-25 keV) X-ray energies, with morphological changes in and around a "loop-top" source associated with a filament eruption observed in EUV and $\mathrm{H} \alpha$. Early appearances and motions of HXR sources have been interpreted as signatures of the formation and development of current sheets (Sui, Holman and Dennis (2004)). In some other events, the motions of footpoints have been correlated with the HXR flux, reflecting the variability of the reconnection rate and the acceleration of electrons (e.g. Krucker et al 2003). But not all flares show a temporal correlation of footpoint or loop motions with HXR flux (Sakao, Kosugi and Masuda (1998); Fletcher and Hudson (2002); Krucker, Hurford and Lin (2003); Grigis and Benz (2005)). It is important to use such observations to test and refine the reconnection model. This flare, because of its long rise phase and multitude of strong, isolated bursts seen in HXR and microwaves, provides additional information on the nature of magnetic reconnection and the association with the acceleration of electrons. 


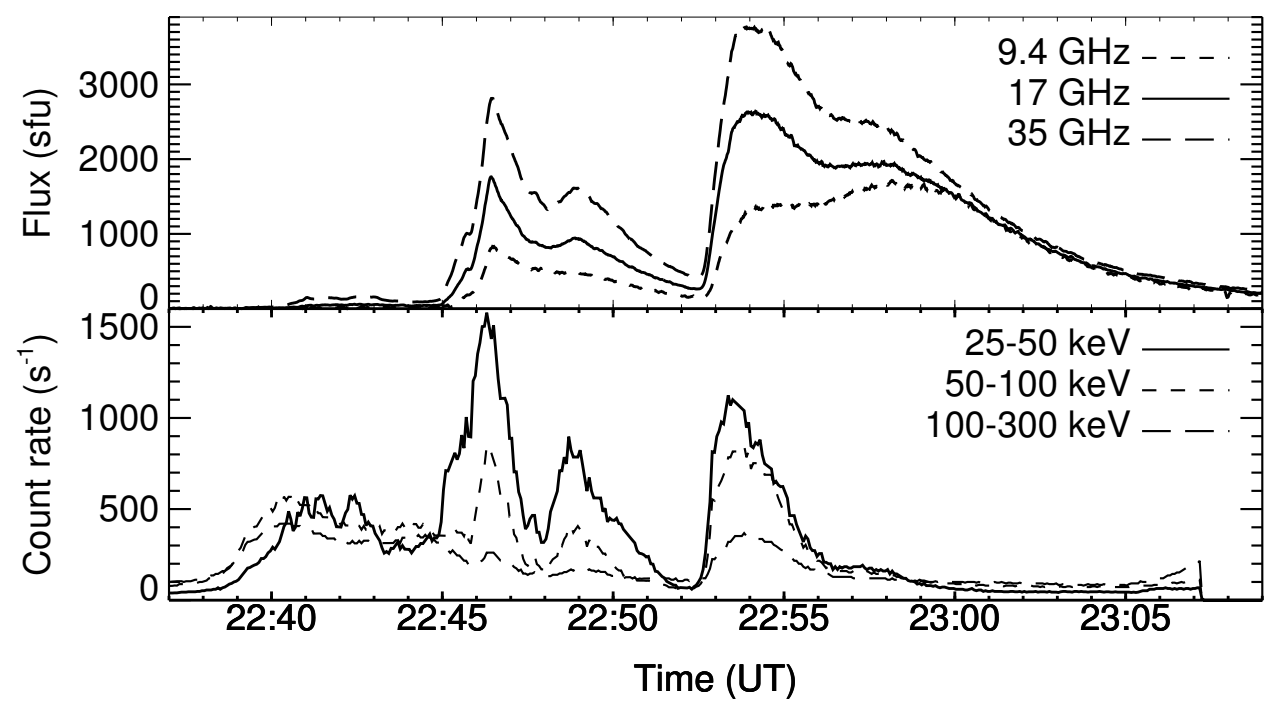

Figure 1. Radio time profiles at 9.4, 17, and $34 \mathrm{GHz}$ from NoRP (upper panel) and hard X-ray count rates at 25-50, 50-100 and 100-300 keV from RHESSI (lower panel).

\section{Observations}

The flare we discuss was a GOES M6.8 event with heliographic position S08E58. Because of Nobeyama night time we could not observe the preflare phase of the event. However the main flare was fully observed at 17 and $34 \mathrm{GHz}$, starting at 22:45 UT. The microwave time profiles (Fig. 1) show the radio burst starting at 22:45 UT and reaching an initial maximum at about 22:46:30 UT. Then there is a minimum at about 22:52:30 UT, followed by a sharp rise to a bigger maximum at about 22:54:00 UT. All the radio light curves are notably smooth, with no structure on short timescales. The 9, 17 and $34 \mathrm{GHz}$ fluxes during the decline after 22:59 UT are similar, suggesting that the radio emission may be thermal at this time. But prior to 22:59 UT the radio spectrum is nonthermal, with a spectral maximum between 9 and $17 \mathrm{GHz}$. At $17 \mathrm{GHz}$ the flare has a flux of order $2700 \mathrm{sfu}$, or more than four times the total flux of the quiet Sun.

Because of the morning hours the beam is elongated at NoRH. Both 17 and $34 \mathrm{GHz}$ sources are located over a neutral line near which a new region appears on MDI magnetogram (Fig. 2) It is possible that the intrusion of this region acted as a trigger for the occurrence of the flare. At all energies greater than $30 \mathrm{keV}$ the RHESSI images show two sources lying on opposite sides of the neutral line.

\section{Preflare activity}

Although the preflare activity was not observed in microwaves due to Nobeyama night time, it was observed well by RHESSI and good images are available from about 22:25 UT until 22:45 UT when the flare was first observed at 17 and $34 \mathrm{GHz}$. The impulsive rise in hard X-rays above $25 \mathrm{keV}$ begins at 22:38 UT (Fig. 1), earlier than in the radio emission, with steepest rise at 22:39 UT toward the first maximum at 22:42 UT (with several sub-peaks in the $25-50 \mathrm{keV}$ range) at energies $25-300 \mathrm{keV}$.

We define the preflare period as occurring earlier than 22:38 UT. Sample low-energy RHESSI images in this period are shown in Fig. 3. The RHESSI images in three energy bands 3-6 (not shown), 6-12 and 12-25 keV ranges are characterized by a compact N-S elongated structure during the interval 22:24-22:37 UT. This is more so at $6-12 \mathrm{keV}$ than at $12-25 \mathrm{keV}$. In the latter case there seems to exist some weak elongated emission north and south of the main strong 

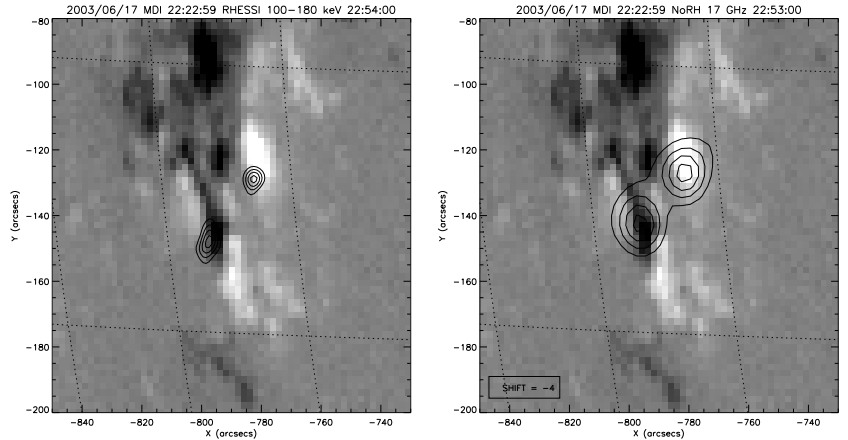

Figure 2. MDI magnetogram at 22:53 UT showing the locations of the 100-180 keV hard X-ray emission (contours, left panel) and $17 \mathrm{GHz}$ microwave emission (contours, right panel). In both cases there are two main sources lying on either side of the neutral line.

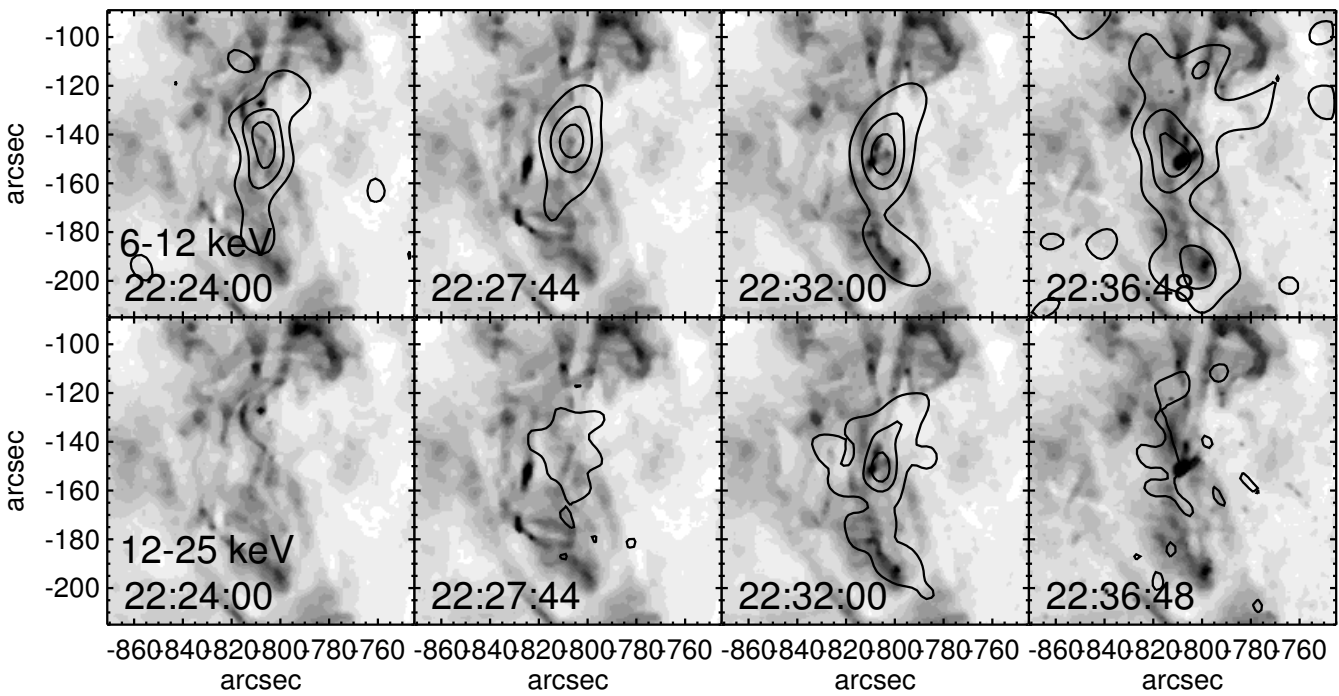

Figure 3. Contours of RHESSI hard X-ray emission over TRACE $195 \AA$ images during the preflare phase. In the upper panels the contours show the $6-12 \mathrm{keV}$ emission and in the lower panels they show the 12-25 $\mathrm{keV}$ emission. The times of the underlying TRACE (inverted) greyscale images match the times of the RHESSI images. In the final panel a very compact brightening can be seen in the $195 \AA$ image at a location just underneath the erupting filament.

compact source oriented in the N-S direction. The photon spectrum between 22:22 and 22:30 appears to be predominantly thermal, changing to power law during the impulsive rise.

A filament over the neutral line in the active region is seen in the TRACE images to be activated during the preflare period. Starting at 22:26 UT, the $195 \AA$ TRACE images show a dark feature moving to the SE of the center of the active region. The dark feature could be tracked until 22:39, after which it became indistinguishable from the background. Its path was approximately linear with $\mathrm{PA}=117$ degrees. Moving bright features paralleled the dark features during the last 2 minutes. The trajectory of the dark feature starts about 20" SE of the 3-12 keV "looptop" HXR source (seen from 22:24 to 22:37 UT), and the track appears to emanate from that source. In addition, a very faint loop lying initially above the filament is seen to erupt outwards at about $900 \mathrm{~km} \mathrm{~s}^{-1}$ in the TRACE images (Fig. 4), starting abruptly at about 22:40 UT when the hard $\mathrm{X}$-rays show their first impulsive rise.

There was a CME associated with this event. According to the SOHO CME catalog, it was a 


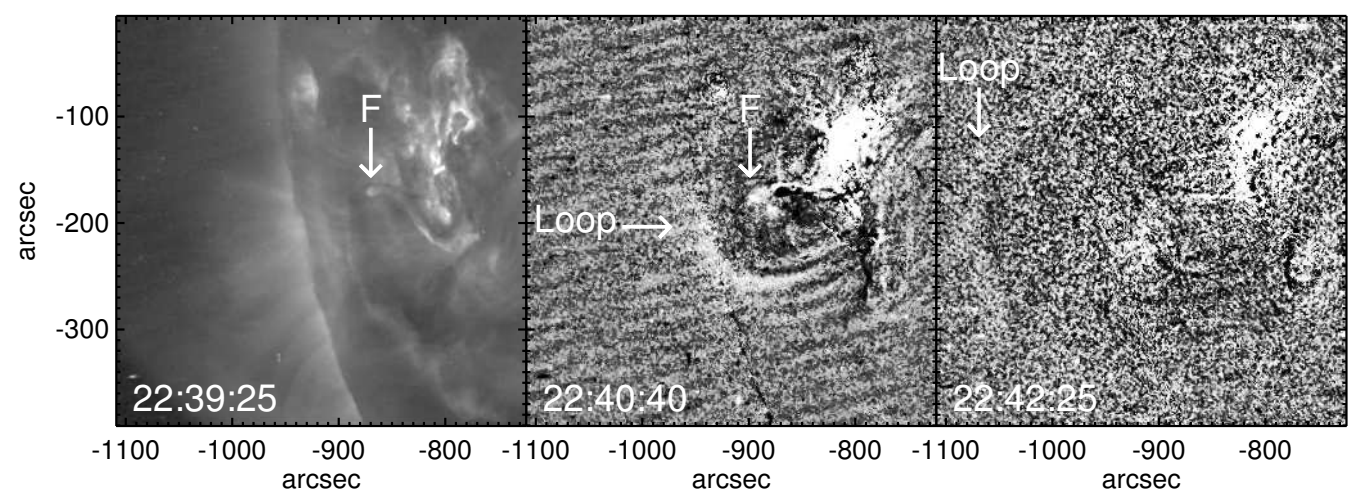

Figure 4. TRACE images of the flaring region at $195 \AA$ during the filament eruption early in the event. The left panel shows the EUV image at 22:39:25 UT: the filament has already started to move upwards and is marked by the arrow. The middle panel shows the difference between the $195 \AA$ images at 22:40:40 and the preceding image at 22:39:25: in addition to the filament this image shows a bright loop-shaped rim surrounding a depression which was not visible at earlier times. The right panel shows a difference image for 22:42:25 minus 22:42:10, when the bright loop has moved to the edge of the field of view (as marked). The mean velocity of this loop over the time between the center and right panels is $900 \mathrm{~km} \mathrm{~s}^{-1}$.

partial halo event in the LASCO C3 field (no C2 data), but its centroid was off the SE limb. The height vs time was quite linear, with a constant speed of $1812 \mathrm{~km} \mathrm{~s}^{-1}$ at PA 117 degrees. The height-time curve extrapolates to the photospheric level at 22:30 UT, approximately the time of the filament lift-off referred to above.

In hard X-rays, preflare features were observed in the 3-6, 6-12 and 12-25 keV bands, but not at higher energies. The 3-6 and 6-12 keV maps are similar during 22:24-22:26 (See Fig. 3 ). Initially, only one component exists at the center of the FOV (the source that the filament structure apparently emerged from). At 22:26 UT, however, a faint secondary source appears about 90 " S of the main source. The secondary source brightens and wanes 3 times between 22:26:00 and 22:32:00.

During the preflare interval, 22:24-22:36, the 12-25 keV maps show the same central source as at lower energies. But in addition, there are other short-lived features that "flicker" around the source. The southern source that appeared in the lower bands at 22:30 also appears at 12-25 keV.

\section{RHESSI Main flare (22:40-23:04 UT)}

At lower energy bands of 6-12 \& $12-25 \mathrm{keV}$, the flaring region is a compact source with apparent maxima at 22:44-22:46 and 22:48-22:50 UT. This continues until the end of the RHESSI flare at about 23:06 UT. Here one does not see footpoints - only the entire unresolved loop. The HXR flare started impulsively above $25 \mathrm{keV}$ at 22:38 UT, attained its first maximum around 22:40-22:42, reached a minor peak at 22:44, and a large peak at about 22:46 UT with a sharper rise time than the earlier phase. After that there is a fourth large peak at 22:49-22:50 UT in the $25-50 \mathrm{keV}$ range. The flux then declines slowly to a minimum at about 22:52 UT. Finally, an intense burst covers the energy range $25-800 \mathrm{keV}$ and starts steeply at 22:52:30 UT in the bands covering $25-300 \mathrm{keV}$.

In the higher energy bands, $25-50$ and 50-100 keV, right from the beginning the main flaring region was resolved into two and sometimes even three component sources (see Fig. 5). Later, at 22:48-22:50 UT, the HXR flaring region has three component sources of which the elongated weak component at 22:48 UT becomes much stronger at 22:49 UT. This component seems to disappear after 22:51 UT, with the northern and middle component remaining as two foot points 


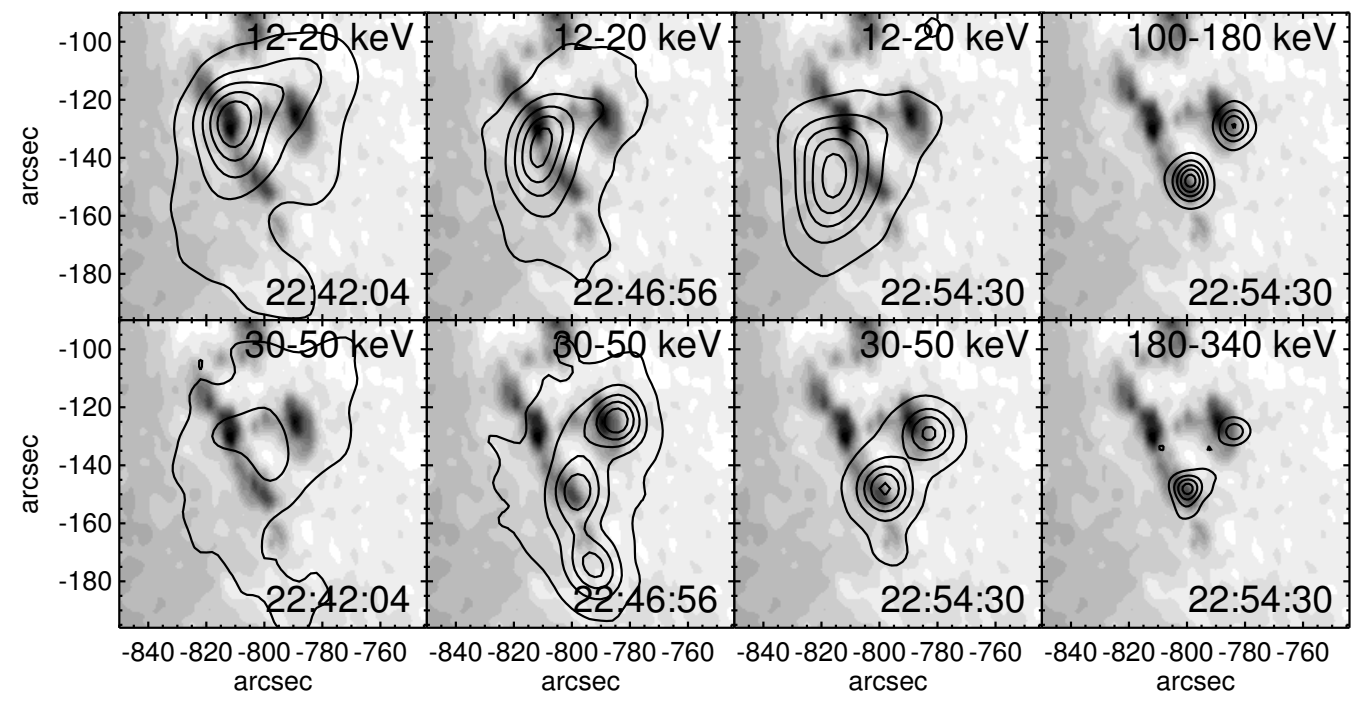

Figure 5. Shows selected RHESSI maps in both low (12-20 keV, upper panels) and higher-energy (30-50 $\mathrm{keV}$, lower panels) bands overlaid on an MDI white-light image of the active region. Contours are at levels $10,30,50,70$ and $90 \%$ of the maximum in each panel except for $30-50 \mathrm{keV}$ at 22:42:04 UT, when fewer contours are plotted. The times correspond to the early phase (22:42:04 UT), first radio peak (22:46:56 UT) and second radio peak (22:54:30 UT). In addition, the two right-most panels show the high energy hard $\mathrm{X}$-ray images at 22:54:30 UT in the 100-180 keV range (upper right) and 180-340 keV range (lower right). These high energy images were made with higher spatial resolution than the 12-20 and 30-50 keV images.

until about 23:02 UT. At 23:03 UT in the lower energy bands we see a distinct HXR loop with one strong component at one foot point, and one sees more of the flaring loop.

Based on the RHESSI count rate plots of 25-800 keV X-rays (Fig. 1), there are five major HXR spikes/acceleration events. Only the last peak, from 22:52:30-22:56:30, shows signifiant emission above $300 \mathrm{keV}$.

\section{NoRH Main Flare}

Fig. 1 shows the NoRP time profiles at 9.4, 17, and $35 \mathrm{GHz}$. The microwave time profiles are characterized by two main broad peaks (which almost look like two separate bursts) with a minimum in between the two bursts. Here we shall describe the time profiles only at 17 and 35 $\mathrm{GHz}$, because these are the NoRH frequencies and we intend to discuss the images at 17 and $34 \mathrm{GHz}$ along with the time profiles. The burst at $17 \mathrm{GHz}$ starts rather steeply at 22:45 with a small hump at 22:45:45 UT, which is followed by a steep rise to a peak at 22:46:30 UT. Then the intensity declines, also rather steeply to a minimum at 22:48 UT. Then it increases again less steeply to a smaller maximum at 22:49 UT. Then there is a slow decline to a pronounced minimum/valley at 22:52:30 UT. From this point the flux rises very steeply to a maximum at 22:54-22:55 UT. This is followed by a less steep decline to a broader hump at 22:57:00-22:58:00 UT. Then there is a gradual decline until the end of the flare at $23: 10 \mathrm{UT}$. At $35 \mathrm{GHz}$ we see the same phenomenon with the exception that the peak at 22:49 UT is much less pronounced and the decline is slower. Further, the pronounced peak (observed at $17 \mathrm{GHz}$ ) is not seen at 35 $\mathrm{GHz}$-only a hump; and instead of the hump (observed at $17 \mathrm{GHz}$ ) at 22:57:00-22:58:00, we see a decent peak at $22: 58 \mathrm{UT}$, followed by a gradual decline as at $17 \mathrm{GHz}$ to the end of the flare at 23:10 UT.

The resolution in the radio images was of order 12 " at $17 \mathrm{GHz}$ and 8 " at $34 \mathrm{GHz}$. At $17 \mathrm{GHz}$ the flaring source at 22:45 UT is a single northern component with weaker features eastward and 
in the southern direction. With time the source becomes elongated in the E-W direction. At 22:46 UT a southern source appears and around the peak time of 22:46:30 UT the southern source becomes more intense. The whole source has the appearance of a NW-S oriented loop. The northern component is polarized in one sense; the weak south component seems to have an oppositely polarized component source around the peak (22:46 UT), lying to the west of the southern I- source. Thus it would seem that the main flaring loop consists of a loop (NW-E) and a second loop oriented E-W. This pattern continues for a minute or so. Ultimately the flaring source evolves into a whole filled loop with a strong $\mathrm{N}-\mathrm{W}$ component and a weaker E-component. At the time of the main minimum ( 22:52:30 UT), $17 \mathrm{GHz}-\mathrm{I}$ shows a simple loop oriented N-S with peaks N \& S of the loop. During the rise phase of the second burst both $\mathrm{N}$ - and S-components intensify,and around the peak time 22:54-22:55 UT the southern component seems to dominate and continues to dominate until the end of the flare. Around the maximum 22:54-22:55 UT, the entire loop has the appearance of two oppositely polarized bipolar loops. Such different polarization structures suggest that multiple loops are involved in the flaring process. Especially around the time of the peak, the two bipolar loops look like interacting loops. Coincidentally after 22:52:30 UT there is an extremely steep rise of intensity to the maximum at 22:54 UT (see the time profiles in Fig. 1). It is tempting to think that this coincidence may be significant; the formation and evolution of the flaring source into several interacting loops may be responsible for this steep rise via magnetic reconnection which produces/accelerates energetic electrons responsible for microwave emission. This interacting loop structure gradually degenerates into a simple loop structure. Starting at 22:45 UT the brightest $34 \mathrm{GHz}$ source is displaced from the brightest $17 \mathrm{GHz}$ position by about 20" to the south (see Fig. 6). This component is strongest at 22:46:30 UT (the first large peak). With time the flaring source develops structures both in the E and S-W directions, and at 22:49 (the time of another peak at $17 \mathrm{GHz}$ ) it becomes a two component source oriented N-E to $\mathrm{S}-\mathrm{W}$. It then evolves and it becomes a largely N-S oriented loop. After the main minimum the 34 $\mathrm{GHz}$ flaring source becomes a loop - similar to the $17 \mathrm{GHz}$ flaring loop - oriented N-W to S-E with a stronger $\mathrm{S}$ - component than the $\mathrm{N}$-component. After this time the $34 \mathrm{GHz}$ flaring source has similar structure and orientation as the $17 \mathrm{GHz}$ source and it continues this pattern until the end time, 23:04 UT.

A type IV-Type II burst was observed during this event in the range of frequencies 30-2000 MHz. A type IV continuum was observed during the period 22:40-23:02 UT from 400-2000 $\mathrm{MHz}$ with the central frequency at around $1000 \mathrm{MHz}$, which is characteristic of many such events. A type II burst was observed to start at 22:45 UT from $300 \mathrm{MHz}$ and ending at 22:53 UT at $100 \mathrm{MHz}$. There was an additional narrow-band continuum centered on 200 MHz, 22:44-23:00 UT. The existence of the type II which represents a shock may be significant, because its onset coincides with the onset of the flare in microwaves. Shock acceleration of microwave- producing electrons may be involved in this case.

In this section we discuss the overlays of RHESSI images in different energy bands on $17 \mathrm{GHz}$ maps. Fig. 7 shows images at 22:53 UT. At higher energies the HXR images agree very well with the 3 -source structure of the radio image, indicating a common origin for the radiations at different wavelengths. However at $12-25 \mathrm{keV}$ the RHESSI source is shifted toward the limb by about 20", most likely due to height difference. We infer that the $12-25 \mathrm{keV}$ image is dominated by thermal material in an arcade of loops, while the higher-energy HXR are nonthermal and arise in footpoints.

During the last large burst (22:53-23:00 UT) close to and around the peak at 22:54-22:55 UT), a $17 \mathrm{GHz}$ central component (south of NW component) dominates, and the strong $17 \mathrm{GHz}$ source lies very close to the HXR strong central component. The N-component is almost co-located with a weaker HXR component - at 22:53-22:54 UT. Gradually the weak $17 \mathrm{GHz}$ component decreases in intensity and merges with the strong south component.

Because of better resolution at higher energies $(>50 \mathrm{keV})$ the HXR flaring sources are re- 


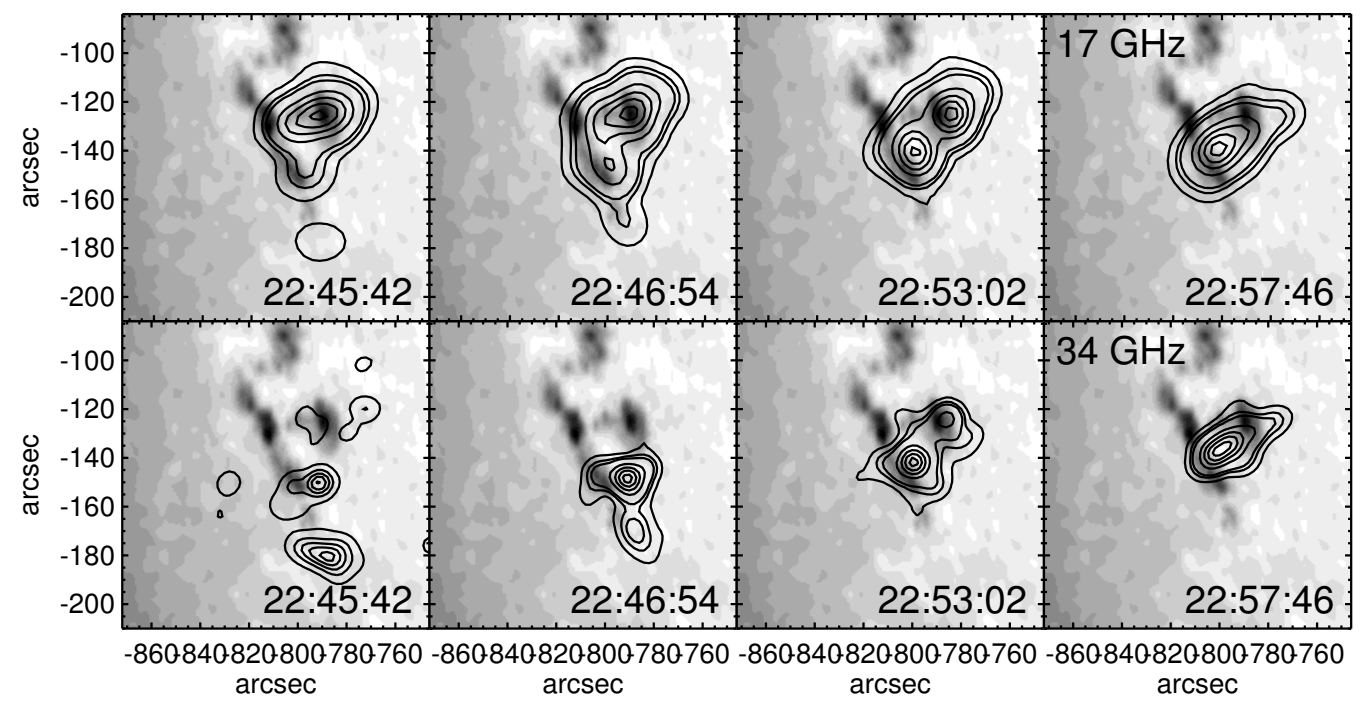

Figure 6. Shows selected NoRH maps at $17 \mathrm{GHz}$ (upper panels) and $34 \mathrm{GHz}$ (lower panels). Contours are at levels 2,6,10,30,50,70 and 90\% of the maximum in each panel except for $34 \mathrm{GHz}$ at 22:45:42 UT, when the lowest contour is at $10 \%$. The times correspond to early in the first impulsive peak (22:45:42 UT), first radio peak (22:46:54 UT), the dip between the two main peaks (22:53:02) and the second radio peak (22:57:46 UT).

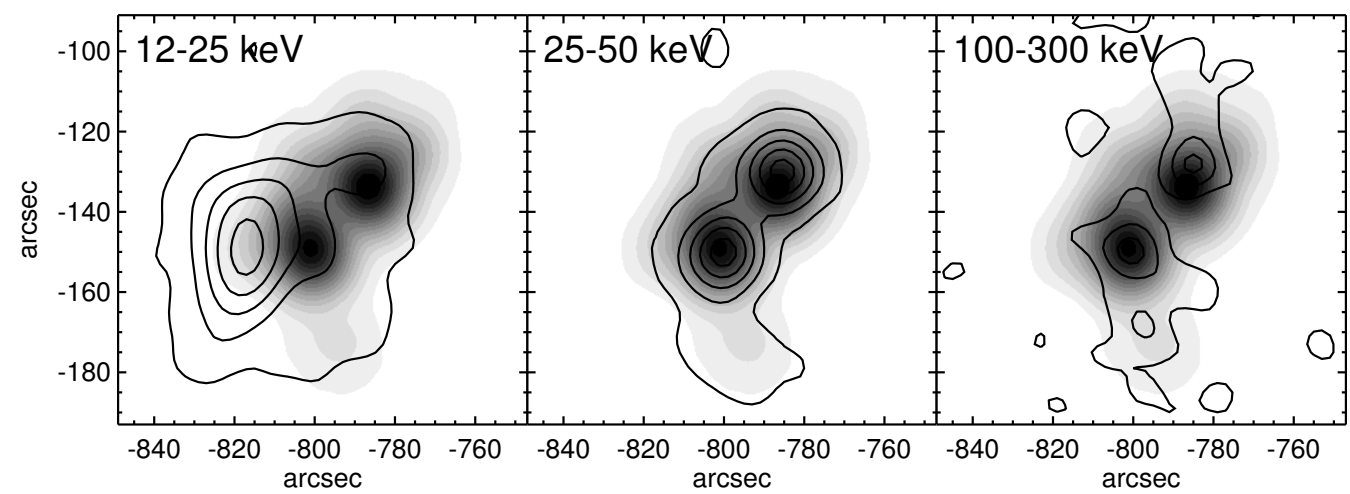

Figure 7. Shows overlays of the RHESSI emission in selected energy ranges over a greyscale image of the $17 \mathrm{GHz}$ emission at 22:53:00 UT. Contours are at levels 10,30,50,70 and 90\% of the maximum in each panel except for $100-300 \mathrm{keV}$ when the lowest contour is at $50 \%$.

solved into several component sources - the overall source oriented N-S. For the first burst the northernmost of these sources is well co-located with the NW $17 \mathrm{GHz}$ component. Two other HXR component sources are also co-located fairly well with the $17 \mathrm{GHz}$ sources around the peak times (22:46-22:47 UT). At the next smaller and broader peak (22:49 UT), the colocation is still good including a southern HXR source co-located with a weak $17 \mathrm{GHz}$ source; further the HXR component sources appear to be foot points of a flaring loop, with an additional NoRH source lying above or in between these two foot point sources. During the 22:53-22:56 burst we have essentially two HXR component sources, very well co-located with two $17 \mathrm{GHz}$ sources. After the peak (22:54-22:55 UT) the northern HXR component decreases in intensity along with the decreasing intensity of the NoRH compt source. Toward the end of the burst, the microwave northern component essentially dies, but the HXR northern component still retains some strength- until the end of the flare, 23:04 UT. 


\section{Summary and Conclusions}

We discuss a flare of GOES class M6.8 using simultaneous imaging observations by RHESSI in HXR and by NoRH in microwaves.

(a) The preflare phase was observed well by RHESSI, but not by NoRH due to Nobeyama night time. The important features of the RHESSI preflare phase is that we observed a TRACE ejecta whose height- time positions were well determined. The trajectory of the absorbing material tracks directly from a 6-25 keV "looptop" source, consistent with the scenario that open field lines extend above a reconnection region near the top of the flare loop, and that material-possibly a plasmoid-is ejected upward from that region. Shortly after the ejection, accelerated electrons are beamed downwards from that reconnection region to the footpoints where they appear in hard $\mathrm{X}$-rays with energies $>25 \mathrm{keV}$.

(b) At 22:45-22:45:30 UT the 6-12 and 12-25 keV image is shifted toward the limb by about 20", most likely due to height difference. The offset diminishes at higher energies $25-50 \mathrm{keV}$ and above where we see at least three component sources which coincide well with the $17 \mathrm{GHz}$ sources. Around the first large peak 22:46:30 UT the flaring source is delineated like a loop better in microwaves than in HXR.

(c) Near the main broad minimum at 22:52:30 we see the flaring loop quite well in microwaves, but less well in HXR. In general,we see both HXR and $17 \mathrm{GHz}$ sources as loops one below the other, with several components in each loop. The strongest component in both seem to coincide well in location.

(d) The interacting multiple loops that develop before 22:54 seem to coincide with the steep rise in intensity to the peak of the 22:53-22:56 burst. We speculate that the second burst steep rise is related to the appearance of two interacting loops. The first burst may be related to the start of the Type II in Hiraiso spectrum - and therefore a shock which accelerates electrons.

(e) The burst maximum at 22:54:30 seems to be produced by two distinct components $-\mathrm{N} \&$ $\mathrm{S}$, eventually with the southern component dominating. In HXR there is a northern extension of the emitting region below $25 \mathrm{keV}$.

$(f)$ The TRACE $195 \AA$ images show, in addition to the filament erupting at a speed of order $100 \mathrm{~km} \mathrm{~s}^{-1}$ or less, a faint loop-shaped feature moving outwards at almost $1000 \mathrm{~km} \mathrm{~s}^{-1}$, initiated at the time of the first abrupt rise in hard X-ray count rates.

(g) This is one of the few events in which we can make hard X-ray images at energies corresponding to microwave-emitting electrons. The 180-340 keV HXR images agree with the microwave images in morphology as we would hope.

\section{Acknowledgements}

Solar research at the University of Maryland is supported by NSF grant ATM 02-33907 and NASA grants NNG 05GM56G, NAG 5-10175, NAG 5-12860, NAG 5-12732 and NAG 5-11872.

\section{References}

Fletcher, L. and Hudson, H.S.: 2002, Solar Phys. 210, 307.

Grigis, P.C. and Benz, A.O.: 2005, Astrophys. J. Letters 625, L143.

Krucker, S., Hurford, G.J., and Lin, R.P.: 2003, Astrophys. J. Letters 595, L103.

Sakao, T., Kosugi, T., and Masuda, S.: 1998, in T. Watanabe and T. Kosugi (eds.), ASSL Vol. 229: Observational Plasma Astrophysics : Five Years of YOHKOH and Beyond, p. 273.

Sui, L., Holman, G.D., and Dennis, B.R.: 2004, Astrophys. J. 612, 546. 